\title{
Femtogram per Milliliter per Gram
}

National Cancer Institute

\section{Source}

National Cancer Institute. Femtogram per Milliliter per Gram. NCI Thesaurus. Code C119347.

A unit of concentration equal to femtogram per milliliter, divided by grams. 